PROCEEDINGS OF THE

AMERICAN MATHEMATICAL SOCIETY

Volume 128, Number 5, Pages 1543-1545

S 0002-9939(99)05316-

Article electronically published on October 6, 1999

\title{
BASIC FORMS FOR TRANSVERSELY INTEGRABLE SINGULAR RIEMANNIAN FOLIATIONS
}

\author{
ROBERT A. WOLAK \\ (Communicated by Christopher Croke)
}

\begin{abstract}
Basic forms for a transversely integrable singular Riemannian foliation with compact leaves are in one-to-one correspondence with "Weyl"invariant differential forms on a generalized section of the foliation.
\end{abstract}

In two recent papers, cf. 3, 4, Peter Michor demonstrated that for a proper isometric action of a Lie group $G$ on a smooth Riemannian manifold admitting a section $S$ the restriction of differential forms induces an isomorphism

$$
\Omega_{h o r}^{p}(M)^{G} \rightarrow \Omega^{p}(S)^{W(S)}
$$

between the space of horizontal $G$-invariant differential forms on $M$ and the space of all differential forms on $S$ which are invariant under the action of the generalized Weyl group $W(S)$ of the section $S$.

The existence of a section assures that on the regular part (the set of points of orbits of the maximal dimension) of the $G$-manifold the orthogonal distribution is completely integrable, i.e. it defines a foliation. In 7] it has been conjectured that the integrability of the orthogonal distribution on the regular part implies the existence of immersed submanifolds which meet orthogonally all orbits. This conjecture has been proved true by $\mathrm{H}$. Boualem in [2] for singular Riemannian foliations (SRF), thus for a larger class of manifolds.

In this short note we propose to demonstrate the following generalization of the Michor theorem for transversely integrable SRF.

Theorem 1. Let $\mathcal{F}$ be a transversely integrable $S R F$ with compact leaves on a compact Riemannian manifold $M$. Let $S$ be a generalized section with the Weyl pseudogroup $\mathcal{W}(S)$. Then the restriction mapping defines an isomorphism $\Omega(M ; \mathcal{F}) \rightarrow$ $\Omega(S)^{\mathcal{W}(S)}$ where $\Omega(M ; \mathcal{F})$ is the algebra of basic forms of the foliated manifold $(M, \mathcal{F})$ and $\Omega(S)^{\mathcal{W}(S)}$ is the algebra of $\mathcal{W}(S)$-invariant forms on the generalized section $S$.

The compactness is used in two places: to prove the existence of a generalized section and in the proof of the surjectivity. In our case (compact leaves) the completeness of the Riemannian metric would be sufficient to prove the existence of a generalized section. We also think that using a more delicate topological argument

Received by the editors July 1, 1998.

1991 Mathematics Subject Classification. Primary 57R30.

Key words and phrases. Singular Riemannian foliation, basic forms.

(C)2000 American Mathematical Society 
the compactness assumption can be replaced by the completeness in the proof of the surjectivity.

\section{Preliminaries}

First, let us recall some results concerning transversely integrable SRF, cf. [2], which will allow us to define the Weil pseudogroup.

Proposition 1. Let $\mathcal{F}$ be a transversely integrable SRF with leaves of compact closure. Through any point of $M$ passes an immersed connected submanifold which meets orthogonally all leaves of $\mathcal{F}$.

Boualem takes a leaf of the horizontal distribution (on the regular part). Owing to a result of Blumenthal-Hebda, cf. [1], this leaf meets any "regular" leaf of $\mathcal{F}$ orthogonally. The idea is to extend this leaf over the singular set of the foliation $\mathcal{F}$. To achieve this Boualem studies a tubular neighbourhood of a singular leaf. It turns out that any connected component of the trace of such a leaf of the horizontal distribution is contained in a fibre of a suitably chosen tubular neighbourhood and moreover, it is a part of the trace of a linear subspace of the fibre. Therefore by taking the whole trace of this linear subspace he was able to extend the leaf. However, it could happen that the extension would intersect itself (it might consist of the trace of several linear subspaces). Therefore the extension of a leaf (of the horizontal distribution) is only an immersed submanifold and it can contain several leaves of the horizontal distribution.

In the regular part any $\mathcal{F}$-leaf curve defines a local isometry between the leaf of $\mathcal{F}^{\perp}$ passing through the starting point of the curve and the leaf passing through the end point of the curve, cf. [1]. Moreover, Boualem proved that any such a local isometry in a suitable small (tubular) neighbourhood of a singular leaf can be extended to a local isometry of generalized sections, cf. Proposition 2.1.1 of [2]. The result is formulated for a very special singular leaf (a point), but obviously it is true for any leaf.

In 6] P. Molino described in detail a new class of SRF called orbit-like foliations. The main characteristic feature of these foliations is the fact that any point $x$ has an adapted neighbourhood in which the foliation is the product of an open neighbourhood of $x$ in the leaf $L_{x}$ passing through this point and the foliation defined by an action of a group of isometries on a suitable vector space (the fibre $N_{x}$ of the normal bundle $N\left(L_{x}\right)=T L_{x}^{\perp}$ at the point $x$ ). This foliation is called the infinitesimal transverse model of $\mathcal{F}$ at $x$. For a suitable small tubular neighbourhood the holonomy transformation defined by a leaf curve beginning and ending in $N_{x}$ can be extended to a global transformation of $N_{x}$. In our case when leaves are compact the traces of leaves of $\mathcal{F}$ on a suitable small ball in $N_{x}$ are the orbits of a compact group of isometries $G_{x}$, whose action generates all holonomy transformations. The restriction of the action of $G_{x}$ to a section generates its Weyl (pseudo)group.

\section{Proof of Theorem 1}

A) The restriction to the chosen section is injective.

The foliation $\mathcal{F}$ is regular on an open and dense subset $M_{0}$ of $M$, cf. [5]. The orthogonal distribution $\mathcal{F}^{\perp}$ on $M_{0}$ is integrable and its leaves are connected components of the trace of generalized sections on $M_{0}$. Any such a leaf intersects all leaves of $\mathcal{F}$ in $M_{0}$. Thus any two basic forms whose restrictions to a leaf of $\mathcal{F}^{\perp}$ (in 
$\left.M_{0}\right)$ are equal are themselves equal on $M_{0}$. By continuity they are equal on the whole manifold $M$.

B) The restriction to the chosen section is surjective.

First let us look at the local problem. Let us choose a point $x$ and let $L$ be the leaf passing through the point $x$. Let $N(L)$ be a suitably chosen small tubular neighbourhood of $L$. Any (generalized) section passing through the point $x$ is of the form $\exp _{x}(W)$ where $W$ is a vector subspace of $T_{x} \mathcal{F}^{\perp}$ and the trace of the foliation $\mathcal{F}$ on $\exp _{x}\left(T_{x} \mathcal{F}^{\perp}\right)=N_{x}$ is given by an action of a Lie group $G_{x}$.

Basic forms on $N(L)$ are in one-to-one correspondence with $G_{x}$-invariant horizontal forms on $N_{x}$. Any section $S_{x}$ of the foliation $\mathcal{F}$ passing through $x$ in $N(L)$ is also a section of the action of the group $G_{x}$. Therefore, according to Michor, basic forms on $N(L)$ are in one-to-one correspondence with $W\left(S_{x}\right)$-invariant forms on $S_{x}$.

The main difficulty in passing from the local case to the global one is the fact that a generalized section can pass many times through a given neighbourhood.

Let $S$ be our generalized section. The trace $M_{0} \cap S$ of $S$ on the regular part $M_{0}$ consists of leaves $\left\{S_{\alpha}\right\}, \alpha \in A$, of the foliation $\mathcal{F}^{\perp}$. The Weyl pseudogroup $\mathcal{W}_{\alpha}$ of any $S_{\alpha}$ consists of local isometries and it is the holonomy pseudogroup $\mathcal{H}_{\alpha}$ of the complete transverse manifold $S_{\alpha}$ of $\left(M_{0}, \mathcal{F}\right)$. Therefore any $\mathcal{W}_{\alpha}$-invariant form $\omega$ can be extended to a basic form $\omega_{0}$ on $M_{0}$. Moreover, the restriction of this form to $\bigcup S_{\alpha}=M_{0} \cap S$ must be $\mathcal{W}(S)$-invariant-directly from the fact that the form $\omega_{0}$ is basic and that the pseudogroup $\mathcal{W}(S) \mid M_{0}$ is generated by holonomy mappings along leaf curves.

It remains to extend the form $\omega_{0}$ over the singular set $\Sigma$. Let $L$ be a leaf in $\Sigma$ and let us choose a component $S_{L}$ of the trace of the generalized section $S$ in a sufficiently small tubular neighbourhood $N(L)$ of $L$. Denote by $\mathcal{W}(L)$ the restriction of the Weyl pseudogroup to $S_{L}$. The form $\omega \mid S_{L}$ is $\mathcal{W}(L)$-invariant. So it can be extended to a basic form $\omega_{L}$ on $N(L)$. On $N(L) \cap M_{0}$ the basic forms $\omega_{0}$ and $\omega_{L}$ coincide, so $\omega_{0} \cup \omega_{L}$ is a smooth basic extension of the form $\omega$. After a finite number of such steps we obtain a global basic form $\tilde{\omega}$ extending the $\mathcal{W}(S)$-invariant form $\omega$. We have just proved the surjectivity of our restriction mapping and thus have completed the proof of the theorem.

\section{REFERENCES}

[1] R. A. Blumenthal, J. Hebda, De Rham decomposition theorem for foliated manifolds, Ann. Inst. Fourier 33(2) (1983), 183-198. MR 84j:53042

[2] H. Boualem, Feuilletages riemanniens singuliers transversalement integrables, Comp. Math. 95 (1995), 101-125. MR 96e:53035

[3] P. Michor, Basic differential forms for actions of Lie groups, Proc AMS, 124,3 (1996), 16331642. MR 96g:57041

[4] P. Michor, Basic differential forms for actions of Lie groups II, Proc AMS 125,7 (1997), 2175-2177. MR 97k:57046

[5] P. Molino, Riemannian Foliations, Progress in Math. 73, Birkhauser (1988). MR 89b:53054

[6] P. Molino, Orbit-like foliations, Proceedings of Geometric Study of Foliations, Tokyo 1993, World Scientific (1994), 97-119. MR 97e:57030

[7] R. S. Palais, C. L. Terng, Critical Point Theory and Submanifold Geometry, Springer LN in Math. 1353, Springer 1988. MR 90c:53143

Instytut Matematyki, Uniwersytet Jagiellonski, Wl. Reymonta 4, 30-059 Krakow, Poland and Wydzial Matematyki Stosowanej Akademia Gorniczo-Hutnicza, al. A. MickIEWICZA, 30 Krakow, Poland

E-mail address: wolak@im.uj.edu.pl 